\title{
Fine mapping the candidate region for peripheral neuropathy with or without agenesis of the corpus callosum in the French Canadian population
}

\author{
Heidi C Howard ${ }^{1,2}$, Marie-Pierre Dubé ${ }^{1,2}$, Claude Prévost ${ }^{3}$, Jean-Pierre Bouchard ${ }^{4}$, \\ Jean Mathieu ${ }^{3}$ and Guy A Rouleau ${ }^{* 1,2}$
}

${ }^{1}$ Centre for Research in Neuroscience, Montreal General Hospital, Montréal, Québec, Canada; ${ }^{2}$ McGill University and the McGill University Health Centre Research Institute, Montreal, Québec, Canada; ${ }^{3}$ Hôtel-Dieu de Chicoutimi, Québec, Canada; ${ }^{4}$ Department of Neurology, Hôpital de l'Enfant Jesus, Quebec, Québec, Canada

Peripheral neuropathy with or without agenesis of the corpus callosum (ACCPN [MIM 2180000]) is an autosomal recessive disease characterised by progressive sensorimotor neuropathy, mental retardation, dysmorphic features and complete or partial agenesis of the corpus callosum. The ACCPN gene was mapped in 1996 to a $4 \mathrm{cM}$ region on chromosome 15. We have since collected additional French Canadian (FC) families and typed a total of 11 polymorphic markers spanning approximately $18 \mathrm{cM}$ on chromosome 15. Through the use of haplotype analysis we have confirmed the presence of a founder haplotype in the FC population, and identified critical recombinants which reduce the ACCPN candidate interval to a $\approx 2 \mathrm{cM}$ or $1000 \mathrm{~Kb}$ region flanked by markers D15S1040 and ACTC. Linkage disequilibrium analysis supports the haplotype data, and suggests that the ACCPN gene lies nearest to marker D15S1232. European Journal of Human Genetics (2002) 10, 406-412. doi:10.1038/sj.ejhg.5200815

Keywords: linkage disequilibrium; agenesis corpus callosum; recombination analysis; founder population; ACCPN

\section{Introduction}

The main clinical features of peripheral neuropathy with or without agenesis of the corpus callosum (ACCPN [MIM 2180000]), consist of a severe progressive sensorimotor neuropathy, mental retardation, dysmorphic features and complete or partial agenesis of the corpus callosum. ${ }^{1}$ Neither the significance nor the causes of the variable involvement of the corpus callosum are known. ${ }^{1,2}$ However, it has been well established that the natural course of the peripheral neuropathy does not vary significantly between individuals with or without agenesis of the corpus callosum. ${ }^{1}$ Signs of ACCPN, such as hypotonia, occur during the first year of life with subsequent delays in motor milestones. During childhood motor function develops slowly, with some patients able to stand or walk with

\footnotetext{
*Correspondence: Guy A Rouleau MD, Ph.D.; L7-224, Montreal General Hospital, 1650 Cedar Ave, Montréal, Québec, H3G 1A4 Canada.

Tel: (514) 934-8094, Fax: (514) 934-8265; E-mail: mi32@musica.mcgill.ca Received 22 January 2002; revised 11 April 2002; accepted 12 April 2002
}

support at 4-6 years of age. ${ }^{3}$ This stage is followed by progressive motor deterioration, often with development of scoliosis. With time, both physical and mental abilities deteriorate, and by adolescence all motor movements are severely impaired and most patients are confined to a wheelchair or bedridden. ${ }^{4}$ ACCPN patients commonly die of complications stemming from respiratory infections during the third or fourth decade of life. ${ }^{3}$ Convulsions are frequent and some patients develop persistent hallucinatory psychosis during adolescence (Prevost C., personel communication). ${ }^{5}$

ACCPN is almost exclusively found in the French Canadian (FC) province of Quebec in an area known as the Charlevoix and Saguenay Lac-St-Jean (CSLSJ) region, where the incidence is $1 / 2117$ and the carrier frequency is $1 / 23{ }^{6}$ Reports of ACCPN patients in other parts of the world are rare and have yet to be confirmed as true ACCPN cases; these reports originate from countries such as Austria ${ }^{7}$, Italy $^{8}$, Spain ${ }^{9}$, and Oman. ${ }^{10}$ Using linkage analysis, Casaubon et $a l^{11}$ initially mapped the ACCPN locus to a $4 \mathrm{cM}$ 
interval on chromosome 15q13-15. We have since collected additional families and tested 11 polymorphic markers on chromosome 15. Haplotype recombination analysis confirms the existence of a founder effect in the FC population and reduces the ACCPN critical interval to a $\approx 2 \mathrm{cM}$ region. Linkage disequilibrium analysis suggests that we begin our search for candidate genes near marker D15S1232.

\section{Subjects, materials, and methods}

Family information

Fifty seemingly unrelated FC ACCPN families, including a total of 231 individuals (67 affected with ACCPN) were identified. Forty families are nuclear, consisting of one to three affected offspring and zero to six unaffected offspring. The remaining 10 families are multinuclear and include $1-$ 3 affected individuals and 4-15 unaffected individuals. After obtaining approval from the appropriate institutional review boards, informed consent was obtained from family members, and blood was collected. DNA was extracted using standard protocol. ${ }^{12}$

\section{Haplotype construction}

Eleven microsatellite markers (Figure 1) spanning a maximum of $18 \mathrm{cM}$ of chromosome 15 were typed in 231 individuals. Marker information was obtained from Généthon, except for markers D15S1232 and ACTC for which the information was obtained from the Cooperative Human Linkage Centre (CHLC) database. Microsatellite marker analysis was performed as described by Casaubon et al. ${ }^{11}$

Haplotypes including all 11 microsatellite markers were manually constructed, using the most parsimonious allele configurations as to minimize the number of recombinants. Haplotypes of chromosomes belonging to affected individuals were compared, and the ACCPN ancestral haplotype was defined as the haplotype bearing the alleles found most frequently in ACCPN patients. Affected haplotypes with divergent segments from the ancestral haplotype were assumed to result from recombination or in rare cases, from marker mutation (when an affected haplotype differed from the founder haplotype only at one marker locus by one repeat sequence with identical flanking markers). The candidate interval was defined

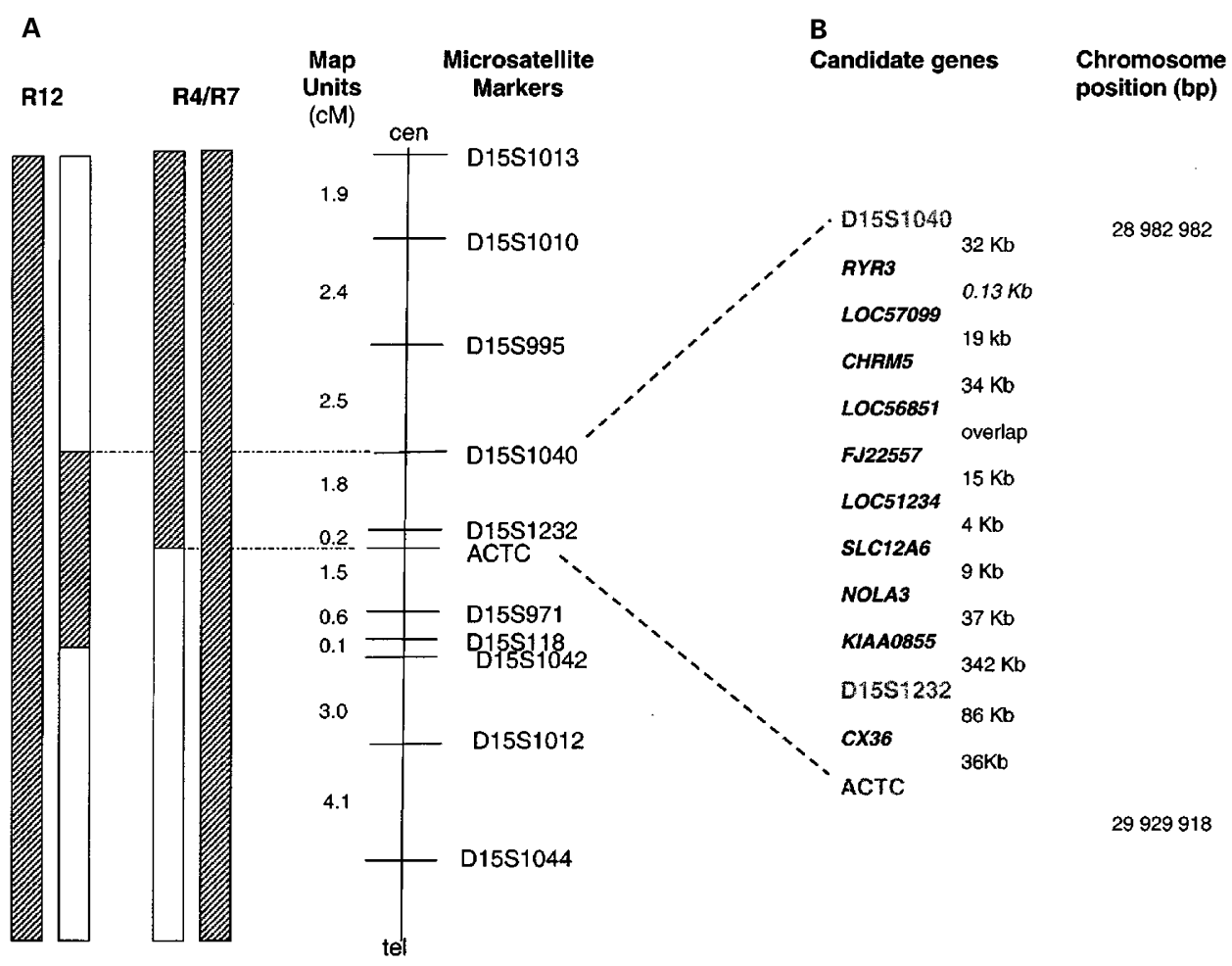

Figure 1 ACCPN critical region on chromosome 15. (A) Genetic map. ACCPN candidate region on chromosome 15q14 as defined by recombinant chromosomes in affected individuals R12, R4, and R7. Inter-marker distances were assigned based on information from Généthon, CHLC, and from physical mapping of the region (unpublished data). Position of critical recombinations are denoted by dotted lines. The shaded regions of the chromosomes represent portions of the haplotypes with ACCPN-associated alleles assumed to originate from one common ancestor. The white portions of the chromosomes indicate haplotypes unrelated to the disease. (B) Physical map. Candidate genes positioned between D15S1040 and ACTC on chromosome 15. Positions and distance approximations between loci (in $\mathrm{Kb}$ ) are based on information obtained from the Human Genome Project at Santa Cruz's working draft of the human genome (December 2000 freeze). 
a

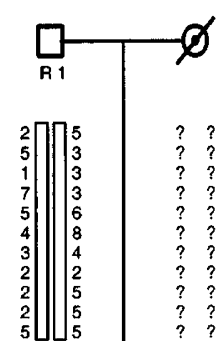

D15S1013 D15S1010 D15S995 Di5S1040
D15S1232

ACTC
D 15971

D15S971

D15S1042

D15S1012

$? ?$

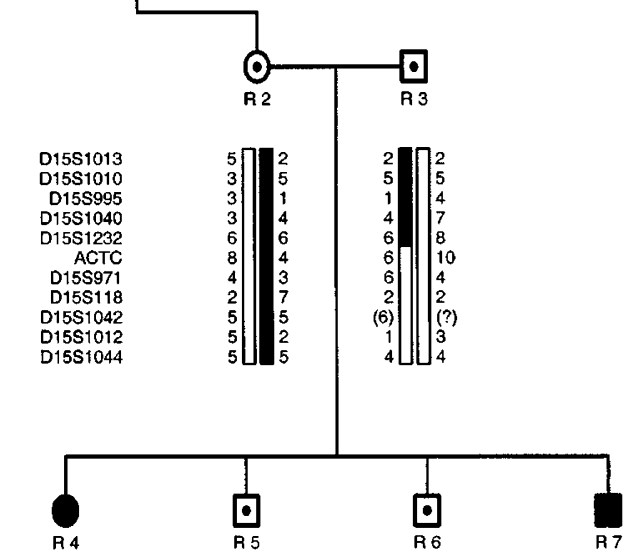

D15S1013 D15S1010 D15S995
D15S1040

D15S1040
D15S1232
ACTC

D15S971

D15S118

D15S1042

D15S1012

D15S1044
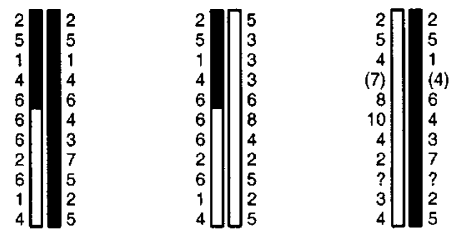

\begin{tabular}{l|l|l}
2 & 2 \\
5 & 5 \\
1 & 4 \\
4 & 1 \\
6 & 6 \\
6 & 6 \\
6 & 6 \\
6 & 4 \\
1 & 3 \\
4 & 7 \\
3 & 5 \\
5 &
\end{tabular}

b

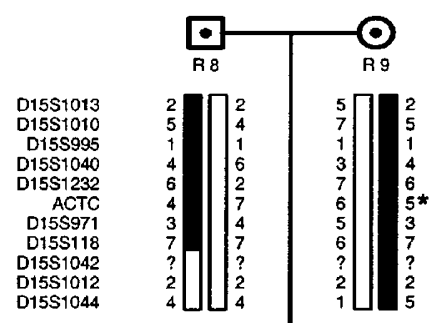

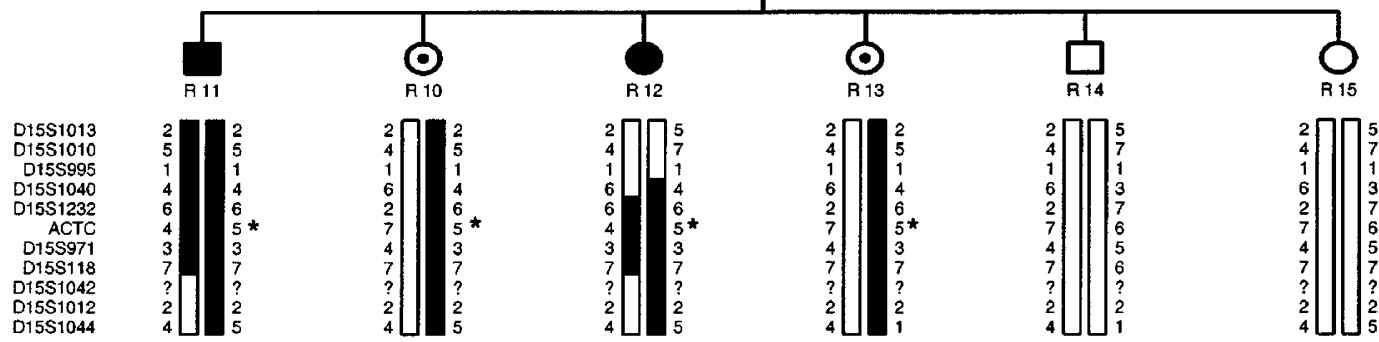


using combined information from historical and familial recombinants.

Linkage disequilibrium analysis

The extent of linkage disequilibrium between markers and the ACCPN phenotype was calculated using the formula: $\mathrm{P}_{\text {-excess }}=\left(\mathrm{P}_{\text {affected }}-\mathrm{P}_{\text {control }}\right) /\left(1-\mathrm{P}_{\text {control }}\right) .{ }^{13}$ One-hundred and five (105) disease haplotypes and 113 control haplotypes from 50 families were considered for the linkage disequilibrium analyses. Affected chromosomes were taken to be the two segregating ACCPN-bearing chromosomes from each pedigree. In families with more than one affected child, the number of ACCPN chromosomes chosen was two, plus the number of ACCPN chromosomes which underwent a familial recombination event. Control chromosomes are the non-transmitted chromosomes of carrier individuals $(n=89)$, and chromosomes of unaffected noncarrier individuals $(n=24)$ in ACCPN families.

\section{Mutation analysis of Cx36}

A total of three control and six ACCPN individuals (including individuals with key recombinant haplotypes which define the ACCPN critical region) were screened by singlestrand conformation polymorphism analysis (SSCP). PCR primers were developed from non-coding sequences flanking exons 1 and 2 of the Cx36 gene such that exonintron boundaries were included in the amplicons. Four sets of primers were developed from exon 2 in order to amplify products with a size range between 200 and 300 base pairs. PCR products were loaded onto non-denaturing polyacrylamide gel (9.5\% acrylamide, 5\% glycerol) as well as onto MDE (mutation detection enhancement) gels. Gels were exposed to autoradiograph film.

\section{Results}

Haplotype analysis and recombination mapping

The most common disease-associated haplotype (4-6-4-3-7) for markers D15S1040-D15S1232-ACTC-D15S971-D15S118, was present in $64 \%(67 / 105)$ of disease chromosomes and none of the control chromosomes. The flanking haplotype 4-6-4 for markers D15S1040-D15S1232-ACTC was present in $71 \%(75 / 105)$ of disease chromosomes, and the haplotype 6-4 for markers D15S1232-ACTC was present in 88\% $(92 /$ 105) of disease-associated chromosomes. Approximately $8 \%$

C

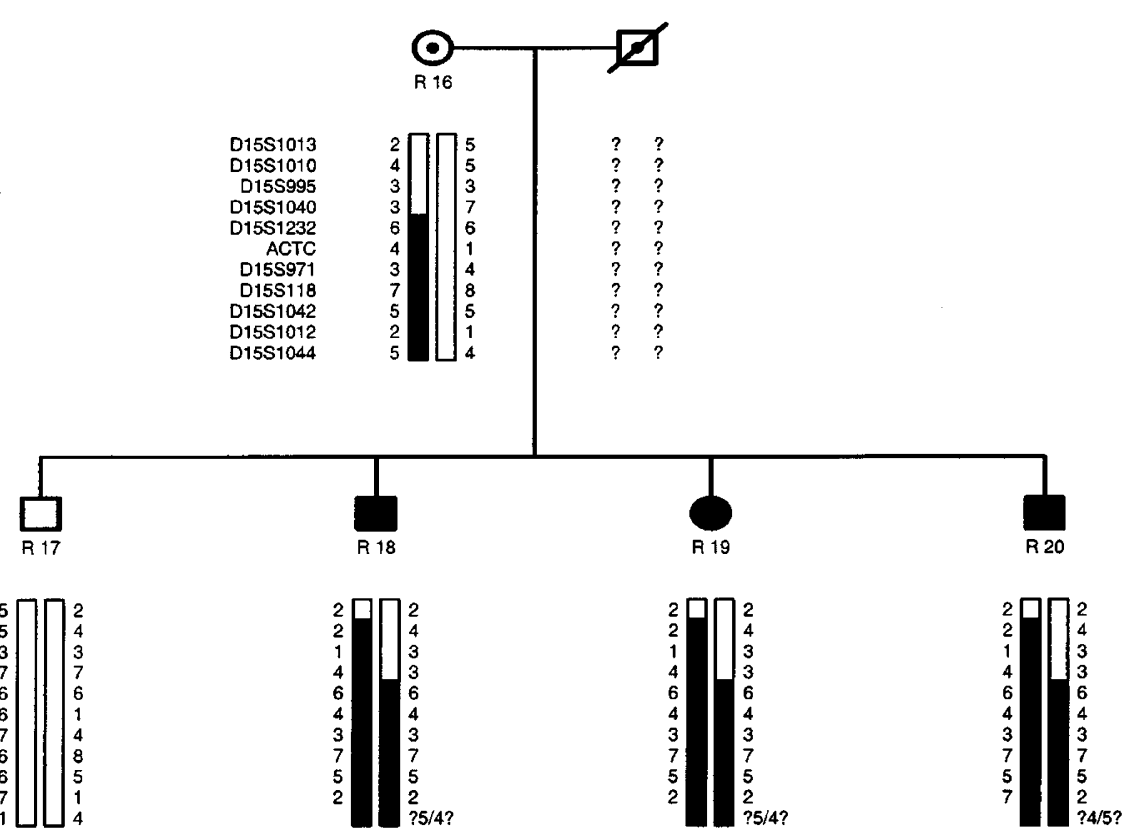

Figure 2 ACCPN pedigrees. Pedigree and haplotype information for 11 markers on chromosome $15 q 14$ in three seemingly unrelated French Canadian families with ACCPN. The founder haplotype (black portion) observed, at least partially, in all affected individuals clearly indicates that these families share a common ancestor. The white portions represent non-disease associated alleles. Question marks placed beside allele numbers denote that the phase of alleles is unknown. Alleles placed within round brackets denote reconstructed genotypes. Question marks placed instead of allele numbers indicate that the genotypes are unknown. (A) Family 1 reveals an ancestral recombination between markers ACTC and D15S1232, which positions the distal boundary of the ACCPN region at ACTC. (B) A paternal recombination in individual R12, positions the proximal limit of the ACCPN region at marker D15S1040. The asterisk denotes a suspected microsatellite mutation for marker ACTC. (C) Family 3 is representative of the additional five families in which an ancestral recombination event between markers D15S1040 and D15S1232 occurred. This supports the conclusion drawn from the familial recombination in family 2 . 
of disease chromosomes retained the same haplotype for all 11 markers: 2-5-1-4-6-4-3-7-5-2-5. This low frequency is not surprising given that the 11 markers span a distance of $18 \mathrm{cM}$.

One ancestral recombination event was observed in one family (Figure 2A) between markers ACTC and D15S1232, placing the ACCPN locus centromeric to ACTC. One familial (Figure 2B, individual R12) and six ancestral recombinants (Figure 2C) were identified in individual families between markers D15S1040 and D15S1232, placing the ACCPN gene telomeric to marker D15S1040. The ACCPN locus therefore lies between markers D15S1040 and ACTC in a region of approximately $2 \mathrm{cM}$. In addition, there are three instances where only the disease associated D15S1232 allele (allele 6) is present on the affected chromosome. Since we confirm the existence of a principal founder haplotype in the CSLSJ population, these chromosomes are assumed to be the result of recombination events on either side of marker D15S1232. This suggests that the ACCPN gene may lie very near to D15S1232.

Six of the disease-associated chromosomes could not be identified clearly as bearing allelic variants issued from recombination of the founder ACCPN haplotype at markers ACTC and D15S1232. We consider the alleles at the markers in question as likely mutations of the alleles present on the founder haplotype since at least two flanking markers on either side of the marker in question have retained ACCPN-associated alleles. Four of these haplotypes involve probable mutation of the tetranucleotide marker D15S1232, where the disease-associated allele 6 (281 bp) is suspected to have expanded by one repeat to allele 7 (285 bp). The four haplotypes cannot be readily related to a recent mutation event by extended haplotype comparison, and the number of mutation events leading to the observed haplotypes could be in the range from one to four events. The estimated mutation rate for marker D15S1232, assuming an exponential population growth rate of $0.8,12$ generations depth and $0.85 \%$ of the carrier population sampled, is 0.00438 to 0.01753 . The two additional haplotypes suspected of allelic mutation at a marker involve an expansion of the disease associated allele $4(227 \mathrm{bp})$ at the dinucleotide repeat marker ACTC, to allele 5 (229 bp). As with marker D15S1232, we cannot determine if one or two distinct mutation events took place. The mutation rate for this marker was calculated to be in the range of $0.00438-0.00877$. Both estimated mutation rate ranges are comparable to reported microsatellite mutation frequencies. ${ }^{14}$

\section{Linkage disequilibrium mapping}

Linkage disequilibrium (LD) of alleles with the disease phenotype was clearly demonstrated by simple inspection of the haplotype data. The strongest association was with allele 6 at marker D15S1232 with a P-excess value of 0.97 . Calculations including presumed mutations at marker D15S1232 result in a P-excess value of 0.92 (Table 1).

\section{Mutation analysis of the $\mathrm{Cx} 36$ gene}

Since the FC ACCPN patients studied share at least a portion of the founder haplotype, we expect to find the same variation in all patients if that variation plays a role in the pathogenesis of the disorder. No variations were detected by SSCP analysis in all six ACCPN patients studied.

\section{Discussion}

The existence of one principal founder haplotype is helpful in order to confirm initial genome scan results, and to

Table 1 Association of alleles on chromosome 15 and the ACCPN phenotype

\begin{tabular}{|c|c|c|c|c|c|}
\hline Markers & $\begin{array}{l}\text { ACCPN } \\
\text { associated } \\
\text { allele }\end{array}$ & $\begin{array}{c}\text { Allele } \\
\text { size } \\
(b p)\end{array}$ & $\begin{array}{l}\text { Affected } \\
\text { allele } \\
\text { frequency }\end{array}$ & $\begin{array}{l}\text { Control } \\
\text { allele } \\
\text { frequency }\end{array}$ & P-excess \\
\hline D15S1013 & 2 & 171 & 0.890 & 0.552 & 0.7544 \\
\hline D15S995 & 1 & 199 & 0.825 & 0.273 & 0.7597 \\
\hline D15S1040 & 4 & 203 & 0.908 & 0.064 & 0.9020 \\
\hline D15S1232 & 6 & 281 & 0.944 & 0.275 & 0.9226 \\
\hline D15S118 & 7 & 229 & 0.835 & 0.163 & 0.8027 \\
\hline D15S1042 & 5 & 196 & 0.797 & 0.351 & 0.6867 \\
\hline D15S1012 & 2 & 162 & 0.719 & 0.449 & 0.4892 \\
\hline D15S1044 & 5 & 195 & 0.409 & 0.193 & 0.2674 \\
\hline \multicolumn{6}{|c|}{ P-excess calculated without the suspected microsatellite mutations: } \\
\hline \multicolumn{3}{|l|}{ **D15S1232 } & 0.981 & 0.275 & 0.9735 \\
\hline
\end{tabular}

Table indicates frequency of ACCPN-associated alleles for 11 markers on chromosome $15 q 14$ in affected and control chromosomes. P-excess values were calculated using the formula: $P_{\text {-excess }}=\left(P_{\text {affected }}-P_{\text {control }}\right) /\left(1-P_{\text {control }}\right)$ where $P_{\text {affected }}$ denotes the observed frequency of the diseaseassociated allele on ACCPN chromosomes and $\mathrm{P}_{\text {control }}$ is the observed frequency of the disease-associated allele on control chromosomes. $P_{-}$ excess values were calculated with and without $\left(^{* *}\right)$ suspected allele mutations for markers D15S1232 and ACTC. 
minimise the candidate interval by identifying recombinant chromosomes. Because the CSLSJ population is relatively young, haplotype analysis and linkage disequilibrium based fine mapping studies could be of limited value since affected individuals carry large segments of chromosomes identical by descent. Despite this possible hindrance, we have reduced the ACCPN candidate interval to less than 2 cM between markers D15S1040 and ACTC. The University of California at Santa Cruz's working draft of the human genome estimates the distance between these two markers to be approximately $1000 \mathrm{~Kb}$. Eleven genes have been positioned within this interval (Figure 1): RYR3, LOC57099, CHRM5, LOC56851, FJ22557, LOC51234, KCC3, NOLA3, KIAA0855, CX36, and ACTC.

Although LD analysis does not provide concrete information regarding the flanking markers of a candidate interval, it can contribute additional information such as where to begin looking for genes within the candidate region. In our case, LD analysis suggests that the ACCPN gene lies especially near D15S1232. Our strategy will, therefore, be to begin by screening genes that lie nearest to marker D15S1232 and which also appear to have some expressional or functional relevance to ACCPN.

Telomeric to marker D15S1232 is the connexin 36 gene (Cx36) which lies $\approx 86 \mathrm{~Kb}$ away. $C x 36$ is a particularly interesting candidate gene; first, because of its proximity to marker D15S1232, and secondly, it codes for a neuronal gap junction protein which is expressed during brain development in the mouse $\mathrm{e}^{15}$ as well as in the adult human brain. ${ }^{16}$ Furthermore, mutations in the connexin 32 gene $(C x 32)$ and in the connexin 31 gene $(C x 31)$, which also code for neuronal gap junction proteins, have been associated with different types of neuropathies; Cx32 with an X-linked form of Charcot-Marie-Tooth disease ${ }^{17}$ and $C \times 31$ with a form sensorineural hearing loss and neuropathy. ${ }^{18}$ Since no variations were detected by SSCP analysis in the $C x 36$ gene in all ACCPN patients studied, we will screen other candidate genes found in the ACCPN critical region.

Just centromeric to marker D15S1232 is KIAA0855 $(\approx 340 \mathrm{~kb}$ away) which codes for a golgi protein called Golgin-67. It is expressed in various human tissues including brain, heart, kidney, and liver and it is thought to be involved in processes such as budding of vesicles or in maintenance of the golgi apparatus. ${ }^{19}$ Although KIAA0855 is the centromeric gene closest to D15S1232, its predicted functions do not make it a convincing cause of ACCPN.

The next most plausible genes potentially responsible for ACCPN are, in our opinion, the NOLA3 gene and the solute carrier family 12 potassium/chloride transporter, member 6 gene (SLC12A6). They are approximately the same distance centromeric to $\mathrm{D} 15 \mathrm{~S} 1232, \approx 380 \mathrm{~Kb}$ and $\approx 390 \mathrm{~Kb}$ respectively. NOLA3 is a member of the small nucleolar ribonucleoproteins gene family which is involved in rRNA processing and modification. ${ }^{20}$ Depletion of the NOLA3 product impairs growth. ${ }^{20}$ The solute carrier family 12 potassium/chloride transporter, member 6 gene, SLC12A6 is one member of a group of four potassium chloride cotransporter genes (KCC1-4). This group, in turn, is a member of a larger cation-chloride cotransporter gene family including the thiazide-sensitive $\mathrm{Na}+\mathrm{Cl}-$ cotransporters as well as the loop diuretics-sensitive $\mathrm{Na}+\mathrm{K}+-\mathrm{Cl}-$ (NKCC) cotransporters. ${ }^{21}$ The SLC12A6 product (Kll3) is expressed in tissues including brain, muscle, kidney and heart. It is thought to be involved in the facilitation of electroneutral passage of ions across the cell's plasma membrane and in cell growth regulation. ${ }^{22}$

Both the ryanodine receptor 3 gene (RYR3), which codes for an intracellular calcium release channel involved in cell signalling $^{23}$ and the muscarinic acetylcholine receptor subtype 5 (CHRM5), which is expressed in the brain as well as in peripheral tissues ${ }^{24}$ could be good functional candidates for a neuronal disorder. However, their relatively distant position from marker D15S1232 makes them poorer positional candidates.

Previously, two separate groups reported linkage of a recessive form of hereditary spastic paraplegia (HSP) with a corpus callosum abnormality to chromosome 15q13q15. ${ }^{25,26}$ Martinez et $a l^{26}$ found a maximum multipoint LOD score of 3.14 at a position $0.36 \mathrm{cM}$ from marker D15S971, and the authors suggest the possibility that ACCPN and HSP are allelic disorders. Shibasaka et $a l^{25}$ however, found a maximum multipoint LOD score of 9.68 at a distance of $1.2 \mathrm{cM}$ telomeric to marker D15S994 (which is $6.9 \mathrm{cM}$ telomeric to D15S971). Both of these HSP candidate intervals are distal to the newly refined ACCPN critical interval. This positional mapping data would suggest that the genes responsible for HSP and ACCPN are in fact two distinct genes. Once the gene responsible for ACCPN is identified, we will be able to address this issue directly.

The work presented in this paper validates both the usefulness of the CSLSJ population and the LD method for disease gene fine mapping. More importantly, it brings us one step closer to identifying the gene responsible for ACCPN. Studying genes involved in agenesis of the corpus callosum is extremely important since it is one of the most common brain malformations found in humans, and it is found at an even greater frequency in children with developmental disabilities. ${ }^{27}$ Therefore, finding the gene responsible for ACCPN could provide valuable information for many other disorders involving agenesis of the corpus callosum.

\section{Acknowledgements}

We would like to thank all the families who participated in this study. This work was supported by 'La Fondation des jumelles Coudés', and the Canadian Institute for Health Research. 


\section{Electronic-Database Information}

Cooperative Human Linkage Center: http://lpg.nci.nih.gov/ CHLC

Généthon: http://www.genethon.fr

Online Mendelian Inheritance in Man (OMIM): http:// www.ncbi.nlm.nih.gov/Omim

University of California at Santa Cruz's working draft of the human genome: http://genome.ucsc.edu

\section{References}

1 Mathieu J, Bedard F, Prevost C, Langevin P: Motor and sensory neuropathies with or without agenesis of the corpus callosum: a radiological study of 64 cases. Can J Neurol Sci 1990; 17: 103 - 108.

2 Larbrisseau A, Vanasse M, Brochu P, Jasmin G: The Andermann syndrome: agenesis of the corpus callosum. Can I Neurol Sci 1984; 11: 257-261.

3 Andermann E: Sensorimotor neuropathy with agenesis of the corpus callosum; in Vinken PJ, Bruyen GW (eds): Handbook of clinical neurology. Amsterdam: North-Holland, 1981; Vol 42: pp. 100103.

4 Andermann F, Andermann E, Joubert $\mathrm{M}$ et al: Familial agenesis of the corpus callosum with anterior horn cell disease: a syndrome of mental retardation, areflexia and paraparesis. Trans Am Neurol Assoc 1972; 97: 242-244.

5 Filteau MJ, Pourcher E, Bouchard RH et al: Corpus callosum agenesis and psychosis in Andermann Syndrome. Archives of Neurology 1991; 48: $1275-1280$.

6 De Braekeleer M, Dallaire A, Mathieu J: Genetic epidemiology of sensorimotor polyneuropathy with or without agenesis of the corpus callosum in northeastern Quebec. Human Genetics 1993; 91: $223-227$.

7 Hauser E, Bittner R, Liegl C, Bernert G, Zeitlhofer J: Occurrence of Andermann syndrome out of French Canada - agenesis of the corpus callosum with neuronopathy. Neuropediatrics 1993; 24: $107-110$.

8 Battistella PA: Occurrence of Andermann syndrome out of French Canada - agenesis of the corpus callosum with neuronopathy [letter]. Neuropediatrics 1993; 24: 239.

9 Gurtubay IG, Yoldi ME, Carrera B et al: Andermann syndrome: presentation of a case. Rev Neurol 1997; 25: 1087-1090.

10 Deleu D, Bamanikar S, Muirhead D, Louon A: Familial progressive sensorimotor neuropathy with agenesis of the corpus callosum (Andermann syndrome): a clinical, neuroradiological and histopathological study. Eur Neurol 1997; 37: 104-109.

11 Casaubon LK, Melanson M, Lopes-Cendes I et al: The gene responsible for a severe form of peripheral neuropathy and agenesis of the corpus callosum maps to chromosome 15q [. Am J Hum Genet 1996; 58: 28-34.
12 Sambrook J, Fritsch E, Maniatis T: Molecular cloning: a laboratory manual; Ford N, Nolan C, Fergusson M, Laboratory CSH (eds). Cold Spring Harbor 1989, pp E3-E4.

13 Lehesjoki AE, Koskiniemi M, Norio R et al: Localization of the EPM1 gene for progressive myoclonus epilepsy on chromosome 21: linkage disequilibrium allows high resolution mapping. Hum Mol Genet 1993; 2: 1229-1234.

$14 \mathrm{Xu} \mathrm{X}$, Peng M, Fang Z: The direction of microsatellite mutations is dependent upon allele length. Nat Genet 2000; 24: 396-399.

15 Gulisano M, Parenti R, Spinella F, Cicirata F: Cx36 is dynamically expressed during early development of mouse brain and nervous system. Neuroreport 2000; 27: $3823-3828$.

16 Condorelli DF, Belluardo N, Trovato-Salinaro A, Mudo G: Expression of Cx36 in mammalian neurons. Brain Research Reviews 2000; 32: $72-85$.

17 De Jonghe P, Timmerman V, Nelis E, Martin JJ, C VB: CharcotMarie-Tooth disease and related peripheral neuropathies. J Peripher Nerv Syst 1997; 2: 370-387.

18 Kelsell DP, Dunlop J, Hodgins MB: Human Diseases: clues to cracking the connexin code?. Trends in Cell Biology 2001; 11: 2-6.

19 Jakymiw A, Raharjo E, Rattner JB et al: Identification and Characterization of a Novel Golgi Protein, Golgin-67. J Biol Chem 2000; 275: $4137-4144$.

20 Pogacic V, Dragon F, Filipowicz W: Human H/ACA small nucleolar RNPs and telomerase share evolutionary conserved proteins NHP2 and NOP10. Mol Cell Biol 2000; 20: 9028-9040.

21 Hiki K, D'Andrea RJ, Furze J et al: Cloning, characterization, and chromosomal location of a novel human $\mathrm{K}+-\mathrm{Cl}-$ cotransporter. J Biol Chem 1999; 274: 10661 -10667.

22 Shen MR, Chou CY, Hsu KF et al: The KCL cotransporter isoform KCC3 can play an important role in cell growth regulation. Proc Natl Acad Sci 2001; 98: 14714-14719.

23 Jeyakumar LH, Copello JA, O'Malley AM et al: Purification and characterization of ryanodine receptor 3 from mammalian tissue. 1998; 273: $16011-16020$.

24 Yamada M, Lamping KG, Duttaroy A et al: Cholinergic dilation of cerebral blood vessel is abolished in M5 muscarinic acetylcholine receptor knockout mice. Proceedings of the National Academy of Sciences, 2001; 98: 14096-14101.

25 Shibasaki Y, Tanaka H, Iwabuchi $\mathrm{K}$ et al: Linkage of autosomal recessive hereditary spastic paraplegia with mental impairment and thin corpus callosum to chromosome 15A13-15. Ann Neurol 2000; 48: $108-112$.

26 Martinez MF, Kobayashi H, Pegoraro E et al: Genetic localization of a new locus for recessive familial spastic paraparesis to $15 \mathrm{q} 13$ 15. Neurology 1999; 53: 50-56.

27 Dobyns W: Absence Makes the Search Grow Longer. Am J Hum Genet 1996; 58: 7-16. 